\title{
Landau Levels and Quasiparticle Spectrum of Extreme Type-II Superconductors
}

\author{
Zlatko Tešanovića ${ }^{a}$ 国 and Pedro D. Sacramento ${ }^{a, b}$ \\ ${ }^{a}$ Department of Physics and Astronomy, Johns Hopkins University, Baltimore, MD 21218, USA \\ ${ }^{b}$ Departamento de Física and CFIF, Instituto Superior Técnico, Av. Rovisco Pais, 1096 Lisboa Codex, Portugal
}

(November 14, 2017)

At high magnetic fields and low temperatures, numerous extreme type-II superconductors exhibit Landau quantization of electronic motion. We present an analytic construction of the quasiparticle spectrum in this regime, based on the highfield expansion. The spectrum is gapless and is separated from the familiar low-field regime by a quantum level-crossing transition. Such low energy excitations should lead to observable effects in thermodynamics and transport.

Recent observations of the de Haas-van Alphen (dHvA) oscillations in many extreme type-II superconducting materials clearly indicate the presence of Landau level (LL) quantization within a high-magnetic field $(H)$, low-temperature $(T)$ "pocket", surrounding the $H_{c 2}(0)$ point in the $H-T$ phase diagram (Fig. 1 ). The size of this pocket in conventional type-II systems (like $\mathrm{Nb}$ ) is expected to be negligible. This is so because, within the BCS theory, the scale of the cyclotron splitting between LLs near $H_{c 2}(0), \omega_{c} \sim \omega_{c 2}(0) \equiv e H_{c 2}(0) / m^{*} c$, is set by the condensation energy, $\sim T_{c 0}^{2} / E_{F}$, and should be much smaller than either the thermal smearing, $\sim T$, or the gap $\Delta(T, H)$, the scale for both being $T_{c 0}$. Additional smearing due to disorder, $\Gamma$, makes this tiny pocket effectively irrelevant. In clean, intrinsically extreme type-II systems, the situation is significantly different. We $d e-$ fine the extreme type-II systems as those with the slope of the upper critical field at $T_{c 0}, H_{c 2}^{\prime} \geq 0.3$ Tesla/Kelvin.2 In such systems $\omega_{c 2}(0)$ is comparable to $T_{c 0}$ and there is large pocket in the phase diagram in which the LL structure within the superconducting phase is well defined, i.e. $\omega_{c}>\Delta(T, H), T, \Gamma$. Numerous superconductors belong to this extreme type-II family: high- $T_{c}$ cuprates, A15's, boro-carbides, many organics, etc. The boundaries of the "extreme" pocket, $H^{*}$ and $T^{*}$, defined by $\omega_{c} \sim \Delta(T=0, H)$ and $\omega_{c} \sim T$, extend to $H$ as low as $H^{*} \sim 50 \% H_{c 2}(0)$ and $T$ as high as $T^{*} \sim 30 \% T_{c 0}$ (Fig. 1). 1

Within this "extreme" pocket the solution to the superconducting problem must fully incorporate the LL structure of the normal state. This leads to a set of Bogoliubov-de Gennes (BdG) equations for the LL quantized quasiparticles in presence of the gap function $\Delta(\mathbf{r})$, describing the Abrikosov vortex lattice 3 . There are two main sources of difficulty in solving these equations. First, the number of LLs involved in the pairing is typically 40-100 and the matrix elements of the gap function between these states, $\Delta_{n m}(\mathbf{q})$, are in general quite complex. A more serious difficulty, however, is that the basis which diagonalizes the BCS Hamiltonian involves combined "rotations" both in the Nambu space and the LL basis. To illustrate why this fact seriously impedes analytic progress we first note that, to the leading order in $\Delta / \omega_{c} \ll 1$, the quasiparticle excitation spectrum near the Fermi surface (FS) can be found exactly: $E= \pm \sqrt{\varepsilon_{n}\left(k_{z}\right)^{2}+\left|\Delta_{n n}(\mathbf{q})\right|^{2}}$. 3 It is tempting to generalize this and conclude that the full solution takes the form: $E= \pm \sqrt{\Sigma_{\bar{n} \bar{n}}^{2}+\left|D_{\bar{n} \bar{n}}\right|^{2}}$, where $\Sigma_{\bar{n} \bar{n}}\left(\mathbf{q}, k_{z}\right)$ and $D_{\bar{n} \bar{n}}\left(\mathbf{q}, k_{z}\right)$ are the normal and pairing self-energies, respectively. The physical meaning of such solution would be that we first rotate the LL basis, $\{n\} \rightarrow\{\bar{n}\}$, and then diagonally pair up these new "normal" quasiparticles. This would lead to a simple description, suitable for applications of our standard theoretical machinery. Unfortunately, this conclusion would be incorrect: beyond the leading order, the full solution cannot be put in the above simple form as the "normal" and "pairing" self-energies cannot be simultaneously diagonalized.5 This leads to a loss of physical transparency and makes it difficult to interpret results in familiar terms. Mpstof the work beyond leading order has been numerical. 3.4 . 6 ,

In this Letter we introduce an analytic approach which allows for a transparent and systematic evaluation of corrections to the leading order results. The basic idea can be illustrated by an example of an interacting Fermi liquid: there various properties are in general very difficult to evaluate. However, as long as we are interested in the low $T$ thermodynamics and low energy transport, these properties can be accurately described by the quasiparticle representation of the excitations near the FS. This leads to the low $T$ thermodynamics which is in one-to-one correspondence with that of non-interacting fermions, but with a modified dispersion relation and a reduced spectral weight. In this paper, we construct such a "quasiparticle" representation of the present problem. Of course, there are no interactions in our case but our aim is similar. The original partition function is replaced by the one of "quasiparticles" whose energies do have the desired form $E= \pm \sqrt{\tilde{\varepsilon}_{n n}^{2}+Z^{2}\left|\tilde{\Delta}_{n n}\right|^{2}}$, and which faithfully represents the low $T$ (or low energy) properties of the original problem. Here $\tilde{\varepsilon}_{n n}\left(\mathbf{q}, k_{z}\right)$ and $\tilde{\Delta}_{n n}\left(\mathbf{q}, k_{z}\right)$ are the renormalized normal and pairing self-energies and $Z$ is the "quasiparticle" renormalization factor. All can be computed to the desired accuracy in $\Delta / \omega_{c}$ from a general expression which we provide. Using this "quasiparticle" representation we derive the following results: first, we evaluate the leading order corrections to "normal" and "pairing" self-energies and find that they are of order $\sim \Delta^{2} / \omega_{c}$ and $\sim \Delta^{3} / \omega_{c}^{2}$, respectively. Therefore, 
for $\Delta / \omega_{c} \ll 1$, the features of the spectrum obtained in the leading order are only moderately affected. In particular, we show that the quasiparticle excitations remain gapless to all orders in perturbation theory until $\Delta / \omega_{c}$ exceeds certain critical value. As $\Delta / \omega_{c}$ increases further $\left(H<H^{*}\right)$, the original LL structure is obliterated by strong LL mixing induced by off-diagonal $\Delta_{n m}$ 's. This exact result shows that the gapless "extreme" pocket is truly a novel state of type-II superconductors and is separated from the gapped low-field mixed phase by a quantum level crossing transition. In this context, we point to an important difference between $3 \mathrm{D}$ and $2 \mathrm{D}$ systems. We next present our numerical results for the excitation spectrum and find that they are in agreement with the analytic results. We hope to inspire experiments which could test our results and provide novel insight into the physics of high-field, extreme type-II superconductors.

Our starting point are the BdG equations:

$$
\begin{array}{r}
E u_{k_{z}, \mathbf{q}, n}^{N}=\varepsilon_{n}\left(k_{z}\right) u_{k_{z}, \mathbf{q}, n}^{N}+\sum_{m} \Delta_{n m}(\mathbf{q}) v_{k_{z}, \mathbf{q}, m}^{N} \\
E v_{k_{z}, \mathbf{q}, n}^{N}=-\varepsilon_{n}\left(k_{z}\right) v_{k_{z}, \mathbf{q}, n}^{N}+\sum_{m} \Delta_{m n}^{*}(\mathbf{q}) u_{k_{z}, \mathbf{q}, m}^{N},
\end{array}
$$

where $\varepsilon_{n}\left(k_{z}\right) \equiv \hbar^{2} k_{z}^{2} / 2 m+n \hbar \omega_{c}-\mu, n$ is the LL index, $\hbar \omega_{c} / 2$ has been absorbed into the chemical potential, $\mu$, $\mathbf{q}$ is the magnetic waveyector and $k_{z}$ is the wavevector along the field direction. $3 \Delta_{n m}(\mathbf{q})=\sum_{j} \Delta_{n, m}^{j}(\mathbf{q})$ is the matrix element of $\Delta(\mathbf{r})=\sum_{j} \Delta_{j}(\mathbf{r})^{8}$ between electronic states $\left(k_{z}, \mathbf{q}, n\right)$ and $\left(-k_{z},-\mathbf{q}, m\right)$. $N$ is the number of LLs which participate in pairing. The solution of the above equations gives the quasiparticle states and the energy spectrum within the superconducting phase.

As one crosses into the superconducting state and $\Delta$ becomes finite, there are both perturbative and nonperturbative effects in Eq. (11). We first isolate the non-perturbative part by noticing that the particle bands ( $u$-bands) with the LL index $n_{1}=n+p$ and the hole bands ( $v$-bands) with the LL index $n_{2}=n-p$ $(p=0, \pm 1, \pm 2, \ldots)$ cross at energies $p \hbar \omega_{c}$ whenever $k_{z}= \pm k_{F n} \equiv \sqrt{2 m\left(\mu-n \hbar \omega_{c}\right)}$, where $k_{F n}$ are the Fermi wavevectors of different quasi one-dimensional LL bands. Additional band crossings take place at energies $(p+1 / 2) \hbar \omega_{c}$ and for $k_{z}= \pm \sqrt{k_{F n}^{2}+m \hbar \omega_{c}}$. At the band crossings near $k_{z}= \pm k_{F n}$ the matrix elements $\Delta_{n+p, n-p}(\mathbf{q})$ act non-perturbatively and must be treated by a degenerate perturbation theory. The same is true for $\Delta_{n+p, n-1-p}(\mathbf{q})$ near $k_{z}= \pm \sqrt{k_{F n}^{2}+m \hbar \omega_{c}}$. The effect of all other matrix elements, $\Delta_{n+p, n(-1)-p^{\prime}}(\mathbf{q})\left(p \neq p^{\prime}\right)$, is purely perturbative and can be computed by an expansion in $\Delta / \omega_{c}$. Near $H_{c 2}$, where $\Delta / \omega_{c} \ll 1$, that effect is small and we can obtain the analytic form for the BCS excitation spectrum near $k_{z}= \pm k_{F n}$ :

$$
E_{n, p}=p \hbar \omega_{c} \pm \sqrt{\varepsilon_{n}\left(k_{z}\right)^{2}+\left|\Delta_{n+p, n-p}(\mathbf{q})\right|^{2}}
$$

where $p=0, \pm 1, \pm 2, \ldots$. A similar expression can be written for the spectrum around $k_{z} \sim \pm \sqrt{k_{F n}^{2}+m \hbar \omega_{c}}$.
Typically, we are interested in the quasiparticle spectrum when we discuss low $T$ (or low energy) properties, i.e. $(T, \omega) \ll \Delta(T, H)<\omega_{c}$. In that case only the quasiparticles near the FS are important, $k_{z} \sim \pm k_{F n}$ and $p=0$, and it suffices to consider only the $E_{n, p=0}=$ $\pm \sqrt{\varepsilon_{n}\left(k_{z}\right)^{2}+\left|\Delta_{n n}(\mathbf{q})\right|^{2}}$ bands. The excitations from other, $p \neq 0$, bands (2) are gapped by energies which are too high, $E_{n, p \neq 0}\left(k_{z} \sim \pm k_{F n}\right) \sim p \hbar \omega_{c}$ and their contribution to the quasiparticle thermodynamics is negligible in the low $T\left(T \ll \Delta(T, H) \ll \omega_{c}\right)$ regime. This is the content of the so-called "diasonal approximation" (DA) for the excitation spectrum. 39

The main issue addressed in this paper is how to go beyond Eq. (2) analytically. As one moves further below $H_{c 2}, \Delta / \omega_{c}$ increases (while still $\ll 1$ ) and the corrections coming from $\Delta_{n+p, n-p^{\prime}}(\mathbf{q})\left(p \neq p^{\prime}\right)$ must be included. A pedestrian perturbation theory, starting from (2), leads to expressions whose physical content is difficult to analyze. The reason is that (2) already involves a full Nambu rotation. Consequently, all other terms generated by the pedestrian perturbation theory are fully Nambu rotated. This makes it impossible to disentangle the "normal" from the "paring" part of the problem. In contrast, our standard theoretical machinery works best when we can neatly separate the normal from the pairing part. We propose here a different method of computing the excitation spectrum. Consider the partition function specified by the solution of BdG equations (11), $Z=\prod_{\omega, \mathbf{q}, k_{z}} \mathcal{Z}\left(i \omega, \mathbf{q}, k_{z}\right)$, where

$$
\mathcal{Z}=\prod_{n} \int d \psi_{n} d \bar{\psi}_{n} \exp \left\{\sum_{n, m} \bar{\psi}_{n}(i \omega \mathcal{I}-\mathcal{H})_{n m} \psi_{m}\right\}
$$

Here the set of Grassman variables $\left\{u_{n}, v_{n}\right\}\left(i \omega, \mathbf{q}, k_{z}\right)$ representing the particle (hole) bands of (11) has been arranged in spinors $\bar{\psi}_{n} \equiv\left(\bar{u}_{n} \quad \bar{v}_{n}\right)$. $\mathcal{I}$ and $\mathcal{H}\left(\mathbf{q}, k_{z}\right)$ are two $2 N \times 2 N$ matrices which are, respectively, a unit matrix and a Hamiltonian whose form is evident from the right hand side of Eq. (11). $\{\omega\}$ is the set of fermionic Matsubara frequencies. Note that $\mathcal{Z}\left(i \omega, \mathbf{q}, k_{z}\right)$ is a determinant of a matrix, $\mathcal{Z}=\operatorname{det}\|i \omega \mathcal{I}-\mathcal{H}\|$. When analytically continued, $E \rightarrow i \omega$, this is precisely the secular determinant of the BdG equations (11). Thus, the solutions for quasiparticle energies in (11) are the zeroes of $\mathcal{Z}\left(i \omega \rightarrow E, \mathbf{q}, k_{z}\right)$.

Within our "extreme" pocket $\left(T \ll \Delta(T, H) \ll \omega_{c}\right)$ the main contributions to $Z$ come from $k_{z} \sim \pm k_{F n}$ and $E_{n, p=0}$ bands. In order to evaluate $\mathcal{Z}\left(i \omega, \mathbf{q}, k_{z} \sim k_{F n}\right)$ we first integrate out all $\psi_{n, p \neq 0}\left(i \omega, \mathbf{q}, k_{z} \sim k_{F n}\right)$ in (3):

$$
\mathcal{Z}=\mathcal{Z}_{p \neq 0} \int d \psi_{n, 0} d \bar{\psi}_{n, 0} \exp \left\{\bar{\psi}_{n, 0}(i \omega \mathcal{I}-\tilde{\mathcal{H}})_{00} \psi_{n, 0}\right\}
$$

$\mathcal{Z}_{p \neq 0}\left(i \omega, \mathbf{q}, k_{z} \sim k_{F n}\right)$ is what one gets from (3) with the $E_{n, p=0}$ band excluded. It describes the contribution to thermodynamics arising from all other bands, $E_{n, p \neq 0}$, by themselves. Since $p \neq 0$ bands are far from the FS, $E_{n, p \neq 0}\left(\mathbf{q}, k_{z} \sim k_{F n}\right) \sim p \hbar \omega_{c}$, such contributions are activated with a large gap, i.e. $\sim \exp \left(-p \hbar \omega_{c} / T\right) \ll 1$, and 
their contribution is very small. Thus, the dominant contribution comes from det $\left\|(i \omega \mathcal{I}-\tilde{\mathcal{H}})_{00}\right\|$. Here $\mathcal{I}_{00}$ is a $2 \times 2$ unit matrix while $\tilde{\mathcal{H}}_{00} \equiv \mathcal{H}^{0}+\mathcal{H}^{\prime}$ is a $2 \times 2$ Nambu matrix. The diagonal elements of $\left\{\mathcal{H}^{0}, \mathcal{H}^{\prime}\right\}\left(i \omega, \mathbf{q}, k_{z} \sim k_{F n}\right)$ are $\left\{ \pm \varepsilon_{n}\left(k_{z}\right), \pm \Sigma_{n n}\left(i \omega, \mathbf{q}, k_{z}\right)\right\}$ while the off-diagonal ones are $\left\{\Delta_{n n}(\mathbf{q}), D_{n n}\left(i \omega, \mathbf{q}, k_{z}\right)\right\}$ and their complex conjugates (c.c.). By itself, $\mathcal{H}^{0}$ determines the spectrum of quasiparticles at the FS (described by $\psi_{n, p=0}$ ) in the DA (2). $\mathcal{H}^{\prime}$ is a correction arising from coupling to other spinor bands, $\psi_{n, p \neq 0}$, with $\Sigma_{n n}$ and $D_{n n}$ being the normal and pairing self-energies, respectively. Since the $p \neq 0$ bands are separated from the FS by $p \hbar \omega_{c}$, these self-energies contain denominators $\sim p \hbar \omega_{c}$ and are small for $\Delta / \omega_{c} \ll 1$. The explicit form is obtained from:

$$
\mathcal{H}^{\prime}=\sum_{p \neq 0} \sum_{p^{\prime} \neq 0} \mathcal{H}_{0 p}(i \omega \mathcal{I}-\mathcal{H})_{p p^{\prime}}^{-1} \mathcal{H}_{p^{\prime} 0}
$$

$\mathcal{H}_{0 p}$ is a $2 \times 2$ Nambu matrix whose diagonal elements are zero and off-diagonal ones $\Delta_{n, n+p}(\mathbf{q})$ and its c.c. The resolvent appearing in (5) is that of the BdG system (11) for $k_{z} \sim k_{F n}$ but with the $p=0$ spinor band (2) excluded.

Up to this point there are no approximations and the expression for $\mathcal{H}^{\prime}$ is formally exact. The problem is that the resolvent for the $p \neq 0$ bands involves inversion of a large matrix. However, for $\Delta \ll \omega_{c}$, the resolvent can be calculated perturbatively, to a desired accuracy in $\Delta / \omega_{c}$. In general, it is useful to first perform the Nambu rotation for the $p \neq 0$ bands to get rid of the degeneracies between the $n+p$ particle and $n-p$ hole bands and then proceed with the perturbative expansion. It turns out that this is not necessary if we are only after the leading order corrections. In this way we obtain:

$$
\begin{gathered}
\Sigma_{n n}=\sum_{p \neq 0} \frac{\left|\Delta_{n, n+p}(\mathbf{q})\right|^{2}}{i \omega+\varepsilon_{n+p}\left(k_{z}\right)} ; D_{n n}= \\
=\sum_{p \neq 0, p^{\prime} \neq 0} \frac{\Delta_{n, n+p}(\mathbf{q}) \Delta_{n+p, n+p^{\prime}}^{*}(-\mathbf{q}) \Delta_{n+p^{\prime}, n}(\mathbf{q})}{\left(i \omega+\varepsilon_{n+p}\left(k_{z}\right)\right)\left(i \omega-\varepsilon_{n+p^{\prime}}\left(k_{z}\right)\right)} .
\end{gathered}
$$

$\Sigma_{n n}\left(i \omega, \mathbf{q}, k_{z}\right)$ and $D_{n n}\left(i \omega, \mathbf{q}, k_{z}\right)$ are the normal and pairing self-energies, respectively. They have a frequency dependence arising from the coupling to the $p \neq 0$ spinor bands through the matrix elements $\Delta_{n, n+p}(\mathbf{q})$. We can view them as self-energies of some "interacting" system and proceed to construct the "quasiparticle" representation of (3). This construction uses a Fermi surface expansion of (6). First, we determine the renormalized "quasiparticle" energies from $\tilde{\varepsilon}_{n n}\left(\mathbf{q}, k_{z}\right)=\varepsilon_{n}\left(k_{z}\right)+\Sigma_{n n}(i \omega=$ $\left.\tilde{\varepsilon}_{n n}\left(\mathbf{q}, k_{z}\right), \mathbf{q}, k_{z}\right)$. To the leading order in $\Delta / \omega_{c}$ this gives:

$$
\tilde{\varepsilon}_{n n}\left(\mathbf{q}, k_{z}\right) \approx \varepsilon_{n}\left(k_{z}\right)+\sum_{p \neq 0} \frac{\left|\Delta_{n, n+p}(\mathbf{q})\right|^{2}}{p \omega_{c}} .
$$

We also evaluate the "quasiparticle" renormalization factor, $Z_{n}^{-1} \equiv 1-\partial \Sigma_{n n} /\left.\partial i \omega\right|_{i \omega=\tilde{\varepsilon}_{n n}}$; to the leading order $Z_{n} \approx 1-\sum_{p \neq 0}\left|\Delta_{n, n+p}(\mathbf{q})\right|^{2} / p^{2} \omega_{c}^{2}$. Similarly, we can compute the "renormalized" shape of the FS. The "renormalized" Fermi velocity is given by:

$$
\frac{\tilde{v}_{F n}(\mathbf{q})}{v_{F n}} \approx 1-\sum_{p \neq 0} \frac{\left|\Delta_{n, n+p}(\mathbf{q})\right|^{2}}{p^{2} \omega_{c}^{2}}
$$

$\tilde{\varepsilon}_{n n}$ and $\tilde{v}_{F n}$ determine "quasiparticle" density of states (DOS) at the FS and their q-dependence describes the "broadening" of LLs by the potential scattering induced through the spatial non-uniformity of $\Delta(\mathbf{r})$. This effect is secondary to the leading pairing-induced "splitting" of LLs in Eq. (2). Finally, the renormalized paring matrix element at the FS is:

$$
\begin{gathered}
\tilde{\Delta}_{n n}(\mathbf{q})=\Delta_{n n}(\mathbf{q})- \\
-\sum_{p \neq 0, p^{\prime} \neq 0} \frac{\Delta_{n, n+p}(\mathbf{q}) \Delta_{n+p, n+p^{\prime}}^{*}(-\mathbf{q}) \Delta_{n+p^{\prime}, n}(\mathbf{q})}{p p^{\prime} \omega_{c}^{2}}
\end{gathered}
$$

This "quasiparticle" construction is not an ordinary perturbation theory. It keeps the "normal" and "paring" self-energies separate by postponing the Nambu rotation until the last step. It can be viewed as a "quasiparticle" representation of the BSC quasiparticle spectrum. Such representation is valid as long as we are interested in excitations near the FS.

The above construction can be carried out to any desired degree of accuracy in $\Delta / \omega_{c}$, although the amount of algebra rapidly increases. Certain general statements, however, can be made. In particular, the excitation spectrum determined by the $\mathrm{BdG}$ equations (11) is gapless in a finite interval $\Delta / \omega_{c} \in\left[0, x_{c}\right]$, where $x_{c} \neq 0$ is some critical value. This property can be demonstrated by noticing first that all $\Delta_{n m}(\mathbf{q})$ have a set of common zeroes whose location in the q-space depends only on whether $n+m$ is evep or odd. These are so-called Eilenberger zeroes, $\left\{\mathbf{q}_{i}^{E}\right\}$. In the leading order (2) the gap is determined by the diagonal matrix elements, $\Delta_{n n}(\mathbf{q})$, and it vanishes at $\mathbf{q}=\mathbf{q}_{i}^{E}$ as well as at numerous other points $\left\{\mathbf{q}_{j}^{n n}\right\}$ within the magnetic Brillouin zone (MBZ). As the matrix elements coupling different $\psi_{n, p}$ 's are turned on, it is clear from (5) that $D_{n n}\left(\mathbf{q}=\mathbf{q}_{i}^{E}\right)$ involves only terms with an odd number of matrix elements $\Delta_{n+p, n+p^{\prime}}(\mathbf{q})$, i.e.

$$
D_{n n} \sim \Delta_{n, n+p} \Delta_{n+p, n+p_{1}} \Delta_{n+p_{1}, n+p_{2}} \cdots \Delta_{n+p_{k}, n} .
$$

Note now that it is impossible to form such a string (10) of odd number of $\Delta^{\prime}$ 's without at least one $\Delta_{n+p^{\prime}, n+p^{\prime \prime}}(\mathbf{q})$ having $n+p^{\prime}+n+p^{\prime \prime}$ even. But such a matrix element also must vanish at same $\left\{\mathbf{q}_{i}^{E}\right\}$ as $\Delta_{n n}(\mathbf{q})$. Consequently, at these points, $\mathbf{q}=\mathbf{q}_{i}^{E}$, the spectrum remains gapless. This statement is correct to all orders in perturbation theory and therefore is exact as long as perturbative expansion itself is well-defined. We expect that the radius of convergence of the perturbativexpansion extends from $\Delta / \omega_{c}=0$ to $\Delta / \omega_{c}=x_{c} \sim 1$. 10 At this critical point, the perturbation theory breaks down due to band crossings between neighboring LL branches. For 
$\Delta / \omega_{c}>x_{c}$ the gaps will open up at $\left\{\mathbf{q}_{i}^{E}\right\}$ signaling the destruction of the LL structure by strong mixing due to increasing $\Delta$. Ultimately, for $\Delta / \omega_{c} \gg x_{c}$, there is a crossover to the low- $H$ regime of mini-gapped states in well-separated vortex cores 3 , as established by Norman et al. 1 . This phenomenon of band crossings and disappearance of gapless excitations has been already noticed in numerical solutions of the BdG equations. 3 Here we elucidate its physical origin through analytic arguments.

We now compare our analytic results with the numerical solution of the BdG equations (11). Such solution is very time consuming in the $3 \mathrm{D}$ case so we have solved (11) for the 2D case. Our analytic results can be adapted to the $2 \mathrm{D}$ case but with one key difference: in a 2D system the FS is a line not a sheet. Consequently, the gapless and "near" gapless points in the MBZ might not be located on the FS. In contrast, the Fermi "sheet" of a LL quantized 3D material contains all of the MBZ. As a result, the gapless character of the excitation spectrum will be less pronounced in 2D systems even though the effects of LL quantization itself are more in evidence there than in quasi 3D materials. This difference between $3 \mathrm{D}$ and 2D systems should lead to observable effects in dHvA oscillations 3 H11 $^{12}$, thermodynamics and transport.

We obtain the spectrum as a function of $\Delta$ for different $n_{c} \equiv \mu / \hbar \omega_{c}$. The eigenvalues are grouped in pairs centered around the LL energies. As $\Delta$ grows, the bands start to broaden until eventually the gap between them goes to zero and the band-crossing occurs. The sequential eigenvalues have a mirror-like shape with the neighboring bands. We fixed $\mu$ such that $\left\{\mathbf{q}_{i}^{E}\right\}$ points remain gapless. In $2 \mathrm{D}$, if $\mu$ is adjusted so that an originally gapless point in the MBZ (2) is on the FS, then the gap opened by the off-diagonal terms should be due to the pairing term and thus $\sim \Delta^{3}$. In general, however, the normal contributions at $\mathbf{q}=\left\{\mathbf{q}_{j}^{n n}\right\}$ gapless points are finite and their gaps will be $\sim \Delta^{2}$. A fit of the normal and pairing self-energy contributions for $\mathbf{q}=\mathbf{q}_{j}^{n n}$ as a function of $\mu$, shows that there is a value of $\mu$ for which the coefficient of the $\Delta^{2}$ term vanishes and therefore close to this point the $\Delta^{3}$ term prevails. This provides a criterion fpr locating the "renormalized" 3D FS at all $\left\{\mathbf{q}_{i}^{E}, \mathbf{q}_{j}^{n n}\right\} .10$

An effective measure of the importance of low energy excitations is the density of states (DOS). The shape of the DOS at low energies is determined by the first and second order zeroes found in the spectrum.12 The exact DOS for small $\Delta$ and DOS within the DA are basically equal since $\Delta / \omega_{c} \ll 1$. There is therefore a large DOS at low energies. As $\Delta$ grows a gap appears in 2D. Yet, if $\mu$ is set to emulate 3D, the DOS remains similar to the DA. As $n_{c}$ grows, the region over which the DOS spreads becomes a smaller fraction of $\Delta$, since the number of zeroes grows with $n_{c}$ and the effect of the off-diagonal perturbations decreases, likely due to large cancellations in (A) In Fig. 1 we show the DOS for $n_{c}=40$ and $N=10$ 触 for the values of $\Delta=0.01,0.5,4$. The energy is rescaled by $\omega_{c}$. The nearly diagonal case, $\Delta=0.01$, and $\Delta=0.5$ appear very similar. They both show a linear dependence in the DOS indicating qualitatively same behavior. The case $\Delta=4$ is qualitatively different. A gap is appearing at low energies and the next band has a small gap to the lowest one indicating the band-crossing is imminent. We can see that as $\Delta$ grows some structure is detected in the shape of the DOS that clearly develops for the $\Delta=4$ case. These results demonstrate that within our pocket $\left(H>H^{*}, T<T^{*}\right)$ the DOS is qualitatively similar to the DA and gapless and nearly gapless points $\left\{\mathbf{q}_{i}^{E}, \mathbf{q}_{j}^{n n}\right\}$ still dominate low energy properties.

Our results have direct and observable consequences for a broad range of thermodynamic and dynamic properties. Specific heat, ultrasound attenuation, STM and other forms of tunneling, and many other phenomena, will exhibit power-law-like behaviors, characteristic of low-energy BCS quasiparticles. All these diagnostic tools can now be used to explore the "extreme" pocket, identify gapless behavior and test our prediction that the highand the low-field regime of type-II superconductors differ in this essential respect.

ZT has benefited from a discussion with Prof. J. R. Schrieffer which served as a significant motivation for this work. The authors thank Prof. S. Dukan for discussions. This research was supported in part by the NSF grant DMR-9415549. PDS acknowledges partial support in the form of a PRAXIS XXI Fellowship.

* Electronic address: zbt@pha.jhu.edu

${ }^{1}$ R. Corcoran et al., Phys. Rev. Lett. 72, 701 (1994); G. Goll et al., Phys. Rev. B 53, 8871 (1996). Refferences to additional experimental work are found below 1 .

${ }^{2}$ Note that, for the effective mass, $m^{*}$, of order of the electron mass, $\hbar \omega_{c}(H=1$ Tesla $) \sim 1.34$ Kelvin.

${ }^{3}$ S. Dukan and Z. Tešanović, Phys. Rev. Lett. 74, 2311 (1995); S. Dukan and Z. Tešanović, Phys. Rev. B 49, 13017 (1994); S. Dukan, A. V. Andreev and Z. Tešanović, Physica C 183, 355 (1991).

${ }^{4}$ M. R. Norman, A. H. MacDonald, and H. Akera, Phys. Rev. B 51, 5927 (1995); H. Akera et al., Phys. Rev. Lett. 67, 2375 (1991).

5 The "normal" self-energy refers to a purely potential scattering induced by finite, non-uniform $\Delta(\mathbf{r})$.

${ }^{6}$ P. Miller and B. L. Györffy, J. Phys. Cond. Matter 7, 5579 (1995).

${ }^{7}$ G. M. Bruun, V. N. Nicopoulos, and N. F. Johnson, suprcon/9608004; V. N. Nicopoulos and P. Kumar, Phys. Rev. B 44, 12080 (1991).

${ }^{8}$ We assume that the self-consistent form of $-(\mathbf{r})$ is known from an independent numerical calculation 3 , 1 (

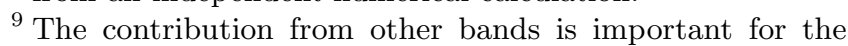
self-consistent determination of $\Delta(\mathbf{r}) .3 .8$

${ }^{10}$ The value of $\Delta$ for which the band-crossing occurs increases with the value of $n_{c}$, since the relevant energy scale goes as 
$\sim \sqrt{2} \Delta /\left(2 \pi n_{c}\right)^{1 / 4}$. 3 G This implies that, for large $n_{c}$, the range of values of $\Delta$ for which the perturbation expansion is adequate is larger.

11 T. Maniv et al., Solid State Comm., 101, 621 (1997).

${ }^{12}$ In $2 \mathrm{D}(3 \mathrm{D})$ the first order zeroes give a linear (quadratic) contribution to the DOS while the second order zeroes give a constant (linear) contribution.
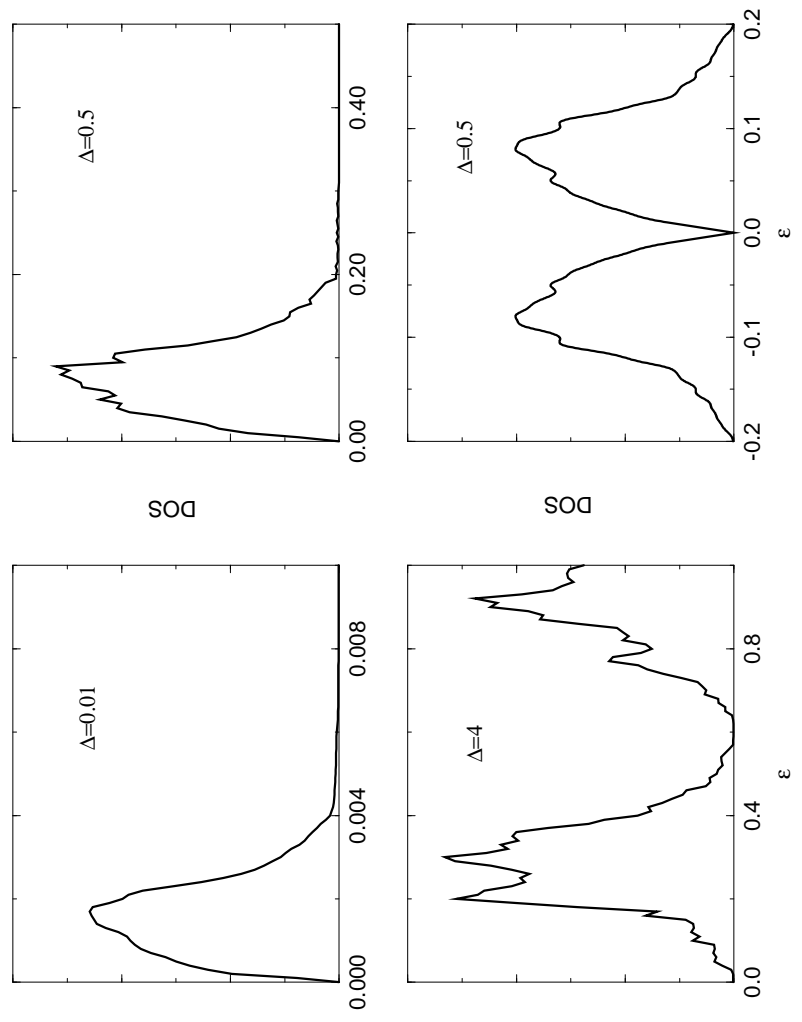

soo

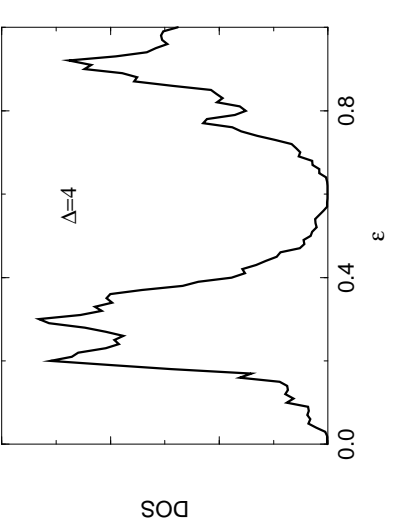

FIG. 1. DOS (in arbitrary units) for several values of $\Delta$ (see text). The inset shows the "extreme" pocket (shaded area). 